\title{
Highly versatile confocal microscopy system based on a tunable femtosecond Er: fiber source
}

\author{
D. Träutlein ${ }^{1}$, F. Adler ${ }^{2}$, K. Moutzouris ${ }^{2}$, A. Jeromin ${ }^{3}$, A. Leitenstorfer ${ }^{2}$, and E. Ferrando-May ${ }^{*}, 1$ \\ ${ }^{1}$ Department of Biology and Center for Applied Photonics, University of Konstanz, 78457 Konstanz, Germany \\ ${ }^{2}$ Department of Physics and Center for Applied Photonics, University of Konstanz, 78457 Konstanz, Germany \\ ${ }^{3}$ Allen Institute for Brain Science, Seattle, WA 98103, USA
}

Received 30 October 2007, accepted 16 November 2007

Published online 27 December 2007

Key words: multiphoton microscopy, femtosecond technology, photomanipulation, three-photon absorption, DNA damage, fluorescent protein

PACS: $42.55 . \mathrm{Wd}$, 82.50.Pt, 87.14.gk, 87.64.mk, 87.64.mm

MESH: H01.181.529.711.200

The performance of a confocal microscopy setup based on a single femtosecond fiber system is explored over a broad range of pump wavelengths for both linear and nonlinear imaging techniques. First, the benefits of a laser source in linear fluorescence excitation that is continuously tunable over most of the visible spectrum are demonstrated. The influences of subpicosecond pulse durations on the bleaching behavior of typical fluorophores are discussed. We then utilize the tunable near-infrared output of the femtosecond system in connection with a specially designed prism compressor for dispersion control. Pulses as short as $33 \mathrm{fs}$ are measured in the confocal region. As a consequence, $2 \mathrm{~mW}$ of average power are sufficient for twophoton microscopy in an organotypic sample from the mouse brain. This result shows great prospect for deep-tissue imaging in the optimum transparency window around $1100 \mathrm{~nm}$. In a third experiment, we prove that our compact setup is powerful enough to exploit even higher-order nonlinearities such as three-photon absorption that we use to induce spatially localized photodamage in DNA.

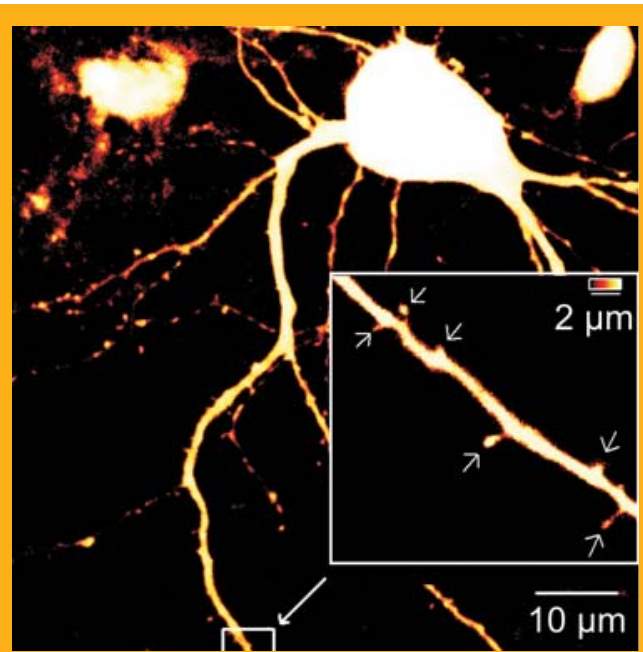

Image of a pyramidal neuron in the CA1 region of the hippocampus acquired by two-photon microscopy

\section{Introduction}

Confocal and multiphoton microscopy $[1,2]$ are two of the most powerful and popular high-resolution imaging techniques in the life sciences. Their range of applications has greatly expanded since the development of genetically encoded fluorescent probes such as GFP and its variants, giving the possibility to visua-

\footnotetext{
* Corresponding author: e-mail elisa.may@uni-konstanz.de, Phone: +49 (0)7531 88 4054. Fax: +49 (0)7531 884033
} 
lize simultaneously multiple components of a living specimen. Recently, a whole new set of yellow and red fluorescent proteins has become available [3, 4]. Thus, multicolor experiments can be performed over the entire visible spectrum. The light sources currently employed for fluorescence excitation in commercially available scanning microscopes are either continuous-wave (cw) lasers emitting at a single defined wavelength for linear confocal microscopy, or femtosecond $\mathrm{Ti}$ : sapphire oscillators providing wavelengths between $700 \mathrm{~nm}$ and $1100 \mathrm{~nm}$ for multiphoton systems. However, the proliferation of multiphoton microscopy is still hampered by the expense of these systems. As an alternative, femtosecond $\mathrm{Cr}$ : forsterite lasers [5] have been employed, but they are not easy to operate due to the poor thermal properties of the gain medium. Moreover, optical parametric oscillators (OPOs) expand the available wavelength range of existing femtosecond lasers $[6,7]$. In terms of stability, compactness and handling, fiber lasers seem to be a perfect irradiation source for microscopy. Third harmonic generation (THG) microscopy [8] has been carried out with femtosecond Er:fiber lasers, which can be built from standard telecommunication components. Sources with Yb-doped gain media provide high average power levels. The disadvantage of these devices as compared to the $\mathrm{Ti}$ :sapphire system is their small tuning range. One approach to extend the spectral coverage is to use supercontinuum generation in photonic crystal fibers (PCFs) [9-13] or tapered fibers [14, 15]. This possibility has been exploited with Ti:sapphire oscillators [10-12, 14] as well as with Yb-based sources $[9,15]$. Due to the necessity to shift the zero-dispersion point of the nonlinear fiber close to the pump wavelength, lasers operating in the region below $1.2 \mu \mathrm{m}$ have to rely on fibers with very small waveguide diameters.

In the present work, we describe a confocal microscope setup for linear and nonlinear imaging equipped with an Er:fiber femtosecond light source that features ultrabroadband emission and short pulse durations at moderate average powers. The entire system is compact, resistant, and produces a highly stable output with excellent noise performance. Furthermore, it is widely tunable in the nearinfrared and visible spectral range.

After characterization of our setup, we present first applications including imaging of cells and tissues expressing the novel red fluorescent proteins mCherry and dTomato as well as the induction of nanoscale DNA damage by multiphoton absorption.

\section{Experimental Setup}

We have built a wide-band excitation source for scanning microscopy consisting of an Er: fiber laser, frequency-conversion schemes and an optimized dispersion management system (see schematic in Figure 1). Its basis is a mode-locked erbium-doped fiber laser oscillator with a single-pass amplifier [16]. After compression to $60 \mathrm{fs}$ employing a silicon prism sequence, it delivers a pulse train at a wavelength of $1.55 \mu \mathrm{m}$ with an average power of $300 \mathrm{~mW}$ at a repetition rate of 107.7 MHz (fundamental). Pulses as short as $200 \mathrm{fs}$ are generated around a wavelength of $775 \mathrm{~nm}$ via frequency doubling in a fan-out poled $\mathrm{MgO}: \mathrm{LiNbO}_{3}$ crystal with an average power of $120 \mathrm{~mW}$ (second harmonic) [17]. Generation of the fourth harmonic in a bismuth borate (BiBO) crystal results in $3 \mathrm{~mW}$ of radiation at a wavelength of $390 \mathrm{~nm}$ [18].

To implement tunable excitation light, the center wavelength of $1.55 \mu \mathrm{m}$ is coupled into a highly nonlinear fiber generating output spectra with two broadband maxima. The spectral shift, and thus the exact wavelength interval covered by the two peaks is controlled by adjusting the chirp of the input pulse [19]. Here, only the short wavelength part is exploited to yield tunable peak wavelengths in the range from $0.98 \mu \mathrm{m}$ to $1.4 \mu \mathrm{m}$ with an average power of $30 \mathrm{~mW}$ (Tunable NIR).

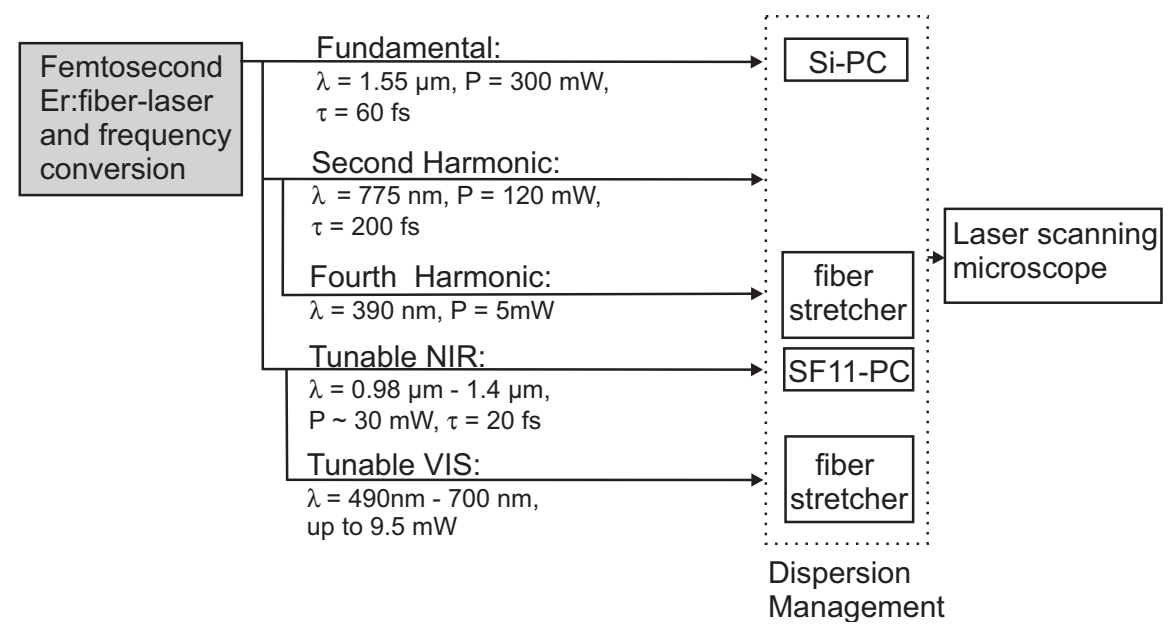

Figure 1 Schematic of the ultrawideband confocal microscopy system: The available wavelengths, power levels, pulse durations and compression strategies are displayed. Si-PC, silicon prism compressor; SF11-PC, SF11 prism compressor. 


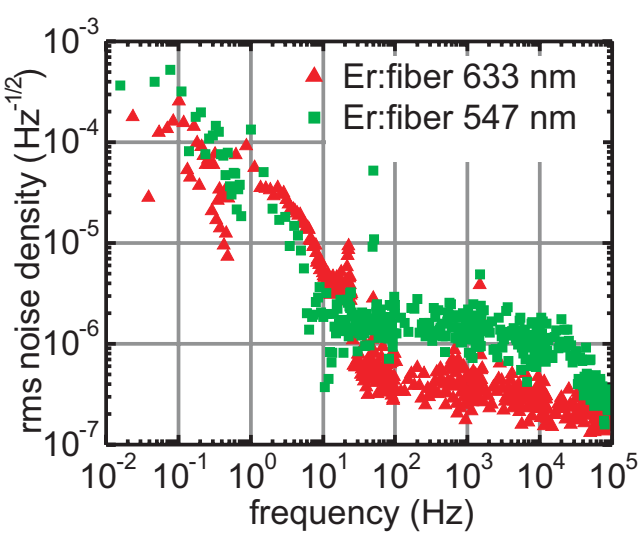

Figure 2 Spectral noise density for the femtosecond fiber laser tuned to two different wavelengths $(547 \mathrm{~nm}$ and $633 \mathrm{~nm})$, rms: root mean square.

Finally, frequency doubling of the tunable NIR in a fan-out poled $\mathrm{MgO}: \mathrm{LiNbO}_{3}$ crystal yields visible radiation from $490 \mathrm{~nm}$ to $700 \mathrm{~nm}$ with an average power as high as $9.5 \mathrm{~mW}$ (tunable VIS) [20].

The excellent noise performance of this setup is demonstrated in Figure 2. Here the spectral noise density is plotted versus frequency. As examples, we display data from the laser tuned to $547 \mathrm{~nm}$ and $633 \mathrm{~nm}$, respectively. The amplitude noise level of the visible radiation is as low as $10^{-6} \mathrm{~Hz}^{-1 / 2}$ in the frequency regime above $20 \mathrm{~Hz}$, despite the fact that it is effectively generated in a nonlinear optical process of $6^{\text {th }}$ order. Consequently, even better noise performance is achieved for other wavelengths where nonlinear conversion of lower order is involved.

Our setup also features a tool to efficiently precompensate the dispersion due to the optical components within the microscope and obtain maximal peak intensities in the focal plane. In the near-infrared, excellent results are obtained with a simple prism compressor. For pulses between $1040 \mathrm{~nm}$ and $1300 \mathrm{~nm}$ (in contrast to the typical Ti:sapphire wavelengths around $800 \mathrm{~nm}$ ), prism materials may be found that not only allow for the compensation of group-velocity dispersion, but also minimize the influence of third-order dispersion. Via numerical simulations, we designed an SF11 compressor with a tip-to-tip distance of $37 \mathrm{~cm}$. This tool delivers extremely short pulse durations (33 fs) in the focal plane that are limited solely by the geometric pulse time delay [21] of the microscope lens.

As we shall see, longer pulses are preferred for linear fluorescence excitation because of less pronounced bleaching. We therefore stretched the pulse length of the tunable visible radiation to 1 ps by selecting an appropriate length of the nonlinear crystal for frequency conversion [20] and subsequent coupling into the microscope via a single-mode fiber (fiber stretcher).
The illumination source presented here offers excitation light for a variety of imaging techniques. The fundamental laser output at $1.55 \mu \mathrm{m}$ may be exploited for THG microscopy $[8,22]$. The second harmonic $(775 \mathrm{~nm})$ is in the spectral range of a $\mathrm{Ti}$ : sapphire oscillator, for which many applications have been described already [23]. The tunable NIR $(0.98 \mu \mathrm{m}$ to $1.4 \mu \mathrm{m})$ can be employed for two-photon excitation of red-shifted dyes whose excitation wavelengths fall beyond the range of Ti:sapphire systems. SHG and THG microscopy have also been demonstrated in this wavelength regime $[24,25]$. The tunable visible radiation offers free choice of excitation wavelength between $490 \mathrm{~nm}$ and $700 \mathrm{~nm}$ for linear confocal microscopy.

The laser was integrated into an LSM 5 Pascal scanning confocal microscope from Carl Zeiss MicroImaging $\mathrm{GmbH}$. The filters and the beam combiner were modified for near-infrared and multiphoton applications. The visible and the ultraviolet are delivered via a single-mode fiber. The infrared wavelengths are coupled in directly via a free-space port.

\section{Results and discussion}

\subsection{Pulse characterization in the focal plane}

Ultrashort light pulses passing through a microscope broaden in time due to the dispersion of filters and lenses. Since nonlinear excitation is influenced by pulse duration, with shorter pulses yielding stronger signals, it is mandatory to characterize the pulse width in the focal plane of the microscope objective. This measurement was performed via second-order interferometric autocorrelation. Two-photon absorp-

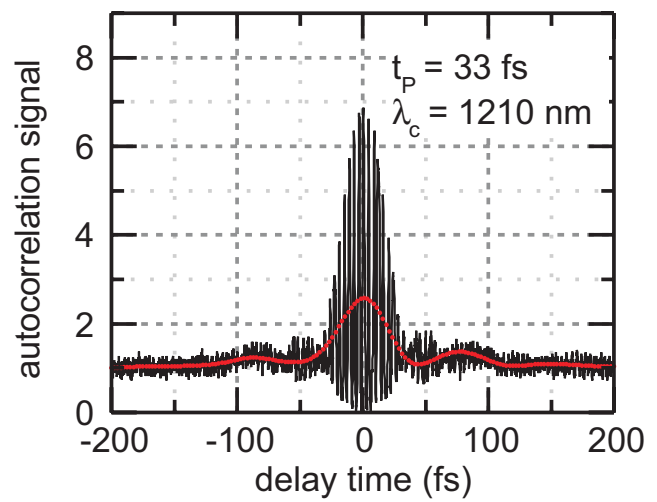

Figure 3 Second-order autocorrelation measured in the focal plane of an Achroplan $40 \times / 0.8 \mathrm{~W}$ objective lens. The figure shows the interferometric autocorrelation (black) and corresponding intensity autocorrelation (red). The retrieved FWHM of the pulse is $33 \mathrm{fs}$. The center wavelength was $1210 \mathrm{~nm}$ with a bandwidth of $110 \mathrm{~nm}$. 


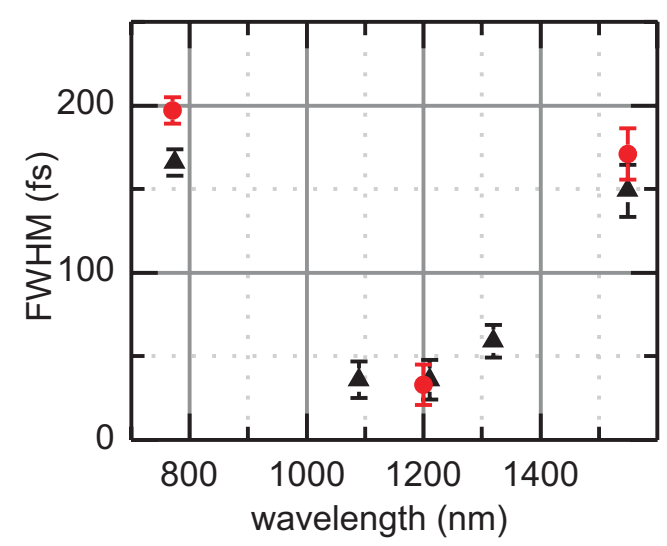

Figure 4 Measured pulse duration (FWHM) in the focal plane versus wavelength for two different objective lenses: Zeiss EC Plan-Neofluar $40 \times / 1.3$ oil objective lens (triangles) and Zeiss Achroplan NIR $40 \times / 0.8 \mathrm{~W}$ objective lens (circles).

tion in direct semiconductor photodiodes serves as the autocorrelation signal [26, 27].

An interferometric autocorrelation trace demonstrating a pulse duration as short as $33 \mathrm{fs}$ at a center wavelength of $1210 \mathrm{~nm}$ measured in the focal plane of a $40 \times$ Zeiss Achroplan NIR objective lens (NA 0.8 , water immersion) is displayed in Figure 3. A similar result was obtained with a $40 \times$ Zeiss EC PlanNeofluar objective lens (NA 1.3, oil immersion). Note that the dispersion of the entire microscope containing filters, beam expander, scan optics and objective lens is effectively compensated up to third order.

Figure 4 shows the wavelength dependence of the pulse durations as measured for the two objective lenses mentioned above. The tunable infrared in-focus pulse width is shorter than $60 \mathrm{fs}$ over the entire wavelength region from $1040 \mathrm{~nm}$ to $1300 \mathrm{~nm}$ due to dispersion compensation with the optimized SF11 prism compressor.

\subsection{Tunable linear excitation with picosecond light pulses in the visible range}

One distinguishing feature of our instrument is that it allows for free selection of excitation wavelength between 490 and $700 \mathrm{~nm}$. Linear confocal imaging in this region is usually performed with fixed $\mathrm{Ar}^{+}$-ion and helium-neon (HeNe) laser lines at $488 \mathrm{~nm}$, $514 \mathrm{~nm}, 543 \mathrm{~nm}, 594 \mathrm{~nm}$, or $633 \mathrm{~nm}$. On the one hand, this fact limits the choice of the fluorophore, on the other hand, it results in many fluorophores being excited in the trailing edge of their absorption spectrum. Therefore, more laser power is required to achieve satisfactory emission signal intensity, thereby increasing background effects, photobleaching, and phototoxicity.
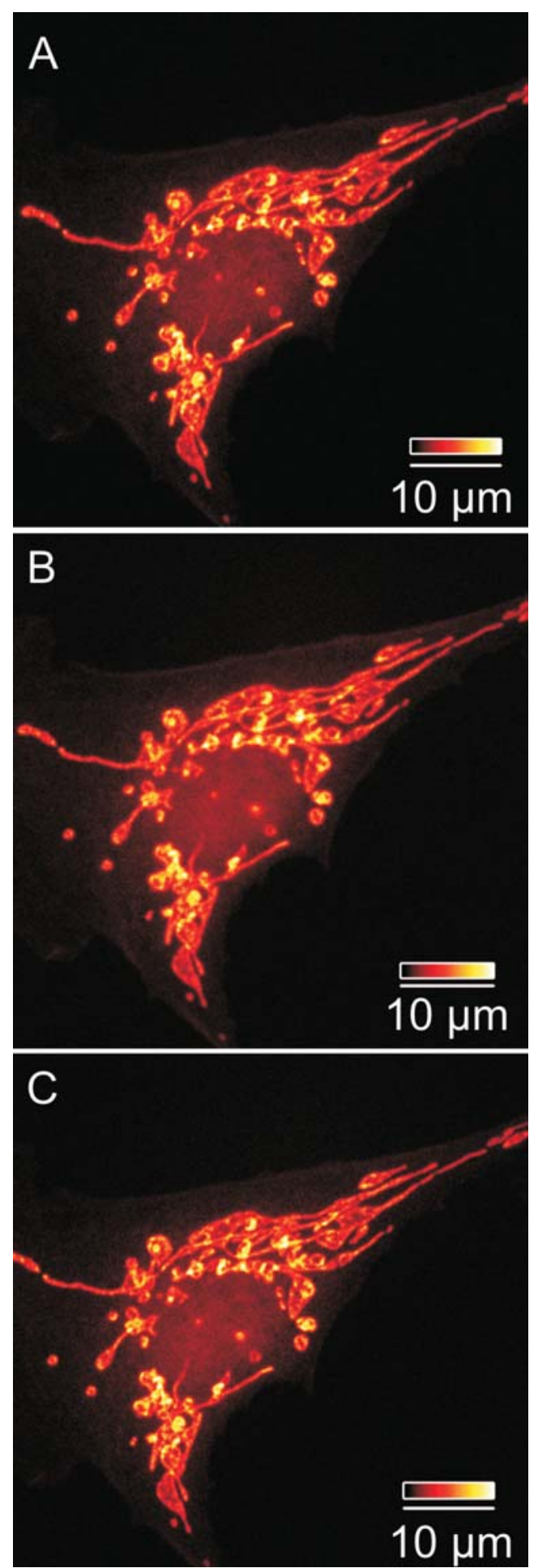

Figure 5 Images of a bovine pulmonary artery endothelial cell (Fluocells \#1, Molecular Probes) obtained under different illumination conditions. Mitochondria are labeled with Mitotracker Red. (A) CW excitation (HeNe) $\lambda_{\mathrm{ex}}=543 \mathrm{~nm}$, $P=40 \mu \mathrm{W}$ (B) and (C) Pulsed excitation, (B) $\lambda_{\mathrm{ex}}=543 \mathrm{~nm}$, $P=44 \mu \mathrm{W},(\mathrm{C}) \lambda_{\mathrm{ex}}=560 \mathrm{~nm}, P=33 \mu \mathrm{W}$. Power levels $(P)$ were measured at the sample. Objective lens: Zeiss EC Plan-Neofluar $40 \times / 1.3$ oil. 
Matching the excitation wavelength to the absorption optimum of a fluorophore might allow reduction of the power of illumination without degradation of signal intensity and image quality. To test this prediction we imaged bovine pulmonary endothelial cells labeled with the mitochondrial dye Mitotracker Red. The maximum of absorption of Mitotracker Red is at $578 \mathrm{~nm}$. It is evident from Figure 5 that the laser power can be reduced by $25 \%$ if the dye is excited at $560 \mathrm{~nm}$ instead of $543 \mathrm{~nm}$ without any loss of information.

In order to compare photobleaching in $\mathrm{cw}$ and pulsed excitation, we recorded the fluorescence emission from HeLa cells expressing mCherry as a function of integrated excitation dose. The results are displayed in Figure 6. We observed a more pronounced bleaching with the Er: fiber laser as compared to the cw-HeNe laser when both systems operated at the wavelength of $543 \mathrm{~nm}$ set by the green $\mathrm{HeNe}$ line and with the same average power of $40 \mu \mathrm{W}$ at the sample. We then tuned the Er:fiber laser to the absorption maximum of mCherry at $587 \mathrm{~nm}$ while keeping the laser power constant. Under these conditions, more molecules are excited as compared to illumination at $543 \mathrm{~nm}$. Quite unexpectedly, we observed a significant decrease of bleaching. This observation was further improved by lowering the laser power to $24 \mu \mathrm{W}$ (same excitation rate as $40 \mu \mathrm{W}$ at $543 \mathrm{~nm}$ ). In this case, the same photobleaching rates were found for $\mathrm{cw}$ - and pulsed-excitation.

The mechanisms underlying photobleaching are quite complex and not fully understood. When comparing the effects of $\mathrm{cw}$ and pulsed excitation it is crucial to keep in mind the relative time scales of the involved processes.

If the main source of photobleaching involves a triplet state, then we would expect similar bleaching

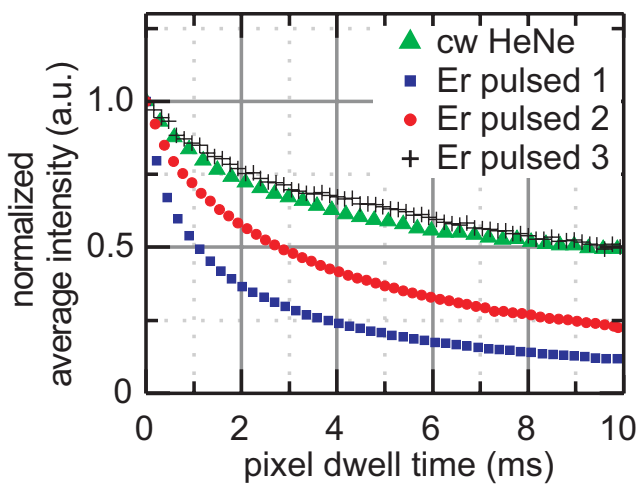

Figure 6 Temporal bleaching behavior observed with HeLa cells expressing mCherry. The average intensity is measured in a region of interest. Legend: cw HeNe, continuous wave helium-neon laser; Er pulsed 1, Er: fiber laser tuned to $543 \mathrm{~nm}$ with $40 \mu \mathrm{W}$ average power at the sample; Er pulsed 2, $587 \mathrm{~nm}$ with $40 \mu \mathrm{W}$; Er pulsed 3, $587 \mathrm{~nm}$ with $24 \mu \mathrm{W}$. Objective lens: Zeiss EC Plan-Neofluar 40/1.3 oil. rates for $\mathrm{cw}$ and pulsed excitation, since the interpulse distance is much shorter than typical triplet lifetimes [28]. In contrast, relaxation from an excited singlet state is in the range of many picoseconds to a few nanoseconds. If the light pulse used for excitation is substantially shorter than the singlet lifetime, as in our experiments, the probability for excited-
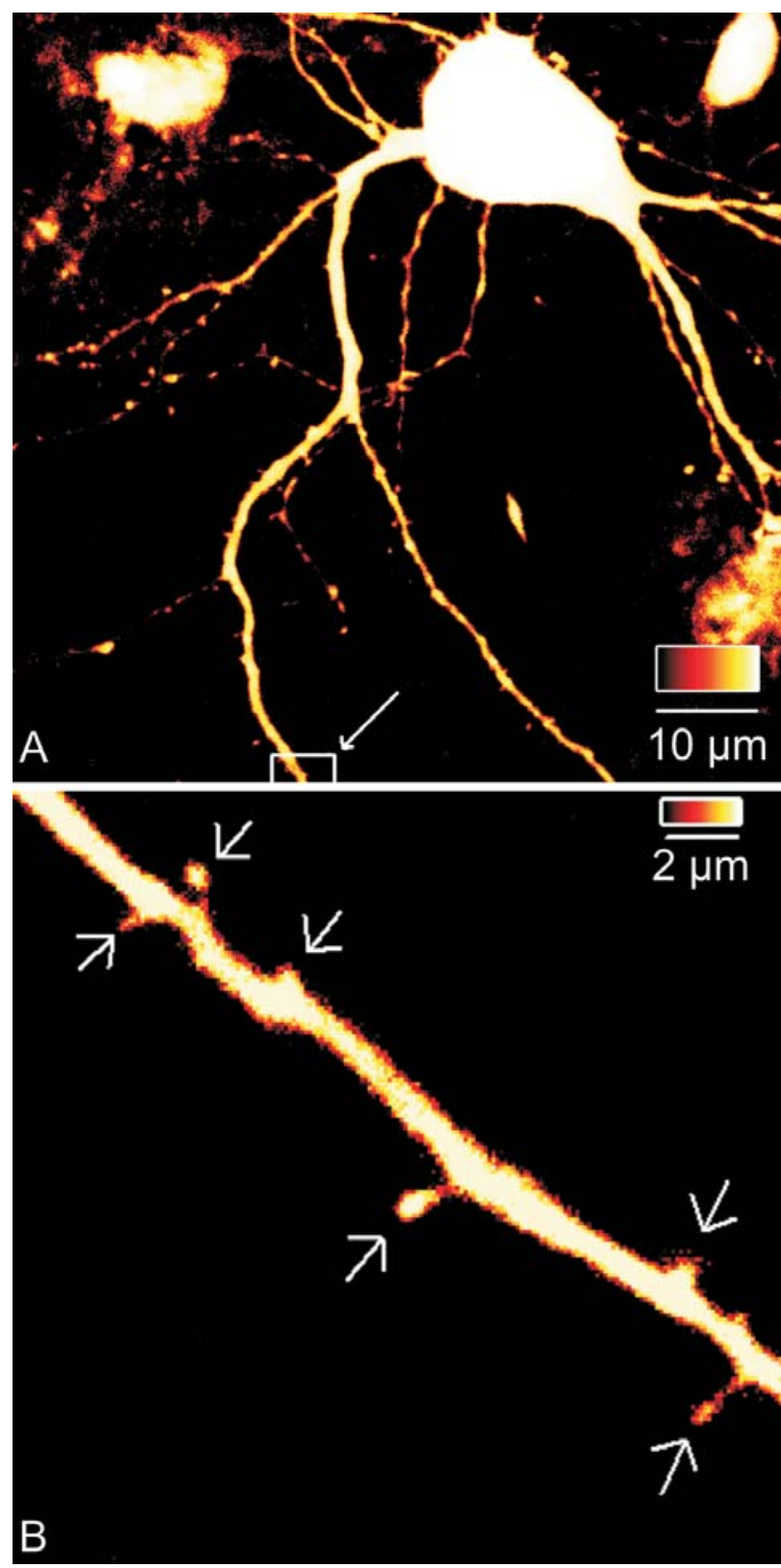

Figure 7 Image of a pyramidal neuron in the CA1 region of the hippocampus acquired by two-photon microscopy. (A) Overview (maximum intensity projection) showing the cell body. (B) Blow up of the region marked in (A). Arrowheads indicate the position of dendritic spines. Excitation power at the sample was $P=3 \mathrm{~mW}$, excitation wavelength $\lambda_{\mathrm{ex}}=1070 \mathrm{~nm}$, pulse duration $t_{\mathrm{P}}=40 \mathrm{fs}$. Objective lens: Zeiss EC Plan-Neofluar $40 \times / 1.3$ oil. 
state absorption into a higher electronic state with high chemical reactivity is significantly enhanced. Therefore, we regard excited-state absorption as the major mechanism of photobleaching in our case.

Spectral variations of excited-state absorption most likely account for the differences that we observed in photobleaching between pulsed excitation at $543 \mathrm{~nm}$ and $587 \mathrm{~nm}$. Based on these considerations we would predict that the bleaching caused by our pulsed laser becomes comparable to a cw source with identical wavelength and average power if the pulses are stretched to durations of the order of the fluorescence lifetime of the fluorophore under examination (e.g. using a highly dispersive fiber). For example, Eggeling et al. [29] found no difference in bleaching between cw and pulsed excitation of rhodamine $6 \mathrm{G}$ with pulses of a duration of $180 \mathrm{ps}$ at a repetition rate of $73 \mathrm{MHz}$.

\subsection{Femtosecond nonlinear excitation in the near infrared}

The tunable femtosecond output in the infrared may be exploited to excite novel fluorophores in complex and turbid tissue samples via two-photon fluorescence microscopy. As an example, we demonstrate two-photon images of organotypic slices from the
CA1 region of the mouse hippocampus (Figure 7). Organotypic slice cultures were focally infected with a modified Sindbis virus [30] expressing dTomato. To our knowledge, these are the first two-photon images of tissue labeled with this new fluorescent protein that has its linear absorption maximum at $554 \mathrm{~nm}$. In the overview image, a cell body and the emanating dendrites are visible. We were able to detect single dendritic spines (Figure 7B), which are small submicrometer membrane protrusions along the dendrites involved in synaptic plasticity. Since the near-infrared excitation wavelength of $1070 \mathrm{~nm}$ exhibits low water absorption and scattering, negligible degradation in spatial resolution was found over the entire sample thickness of $60 \mu \mathrm{m}$. Unexpectedly, only $2 \mathrm{~mW}$ of laser power at the sample were sufficient to obtain high-resolution two-photon images. This finding is due to the extremely short pulse durations achieved by the optimized dispersion compensation. We want to emphasize that these images were recorded via descanned detection. Using a nondescanned detection pathway, as normally employed in two-photon microscopes, will allow further reduction of excitation power.

The high efficiency of our system in nonlinear photochemistry is demonstrated by the generation of DNA lesions with high spatial resolution. DNA has a strong linear absorption band in the UV region with a maximum around $260 \mathrm{~nm}$. The main defects
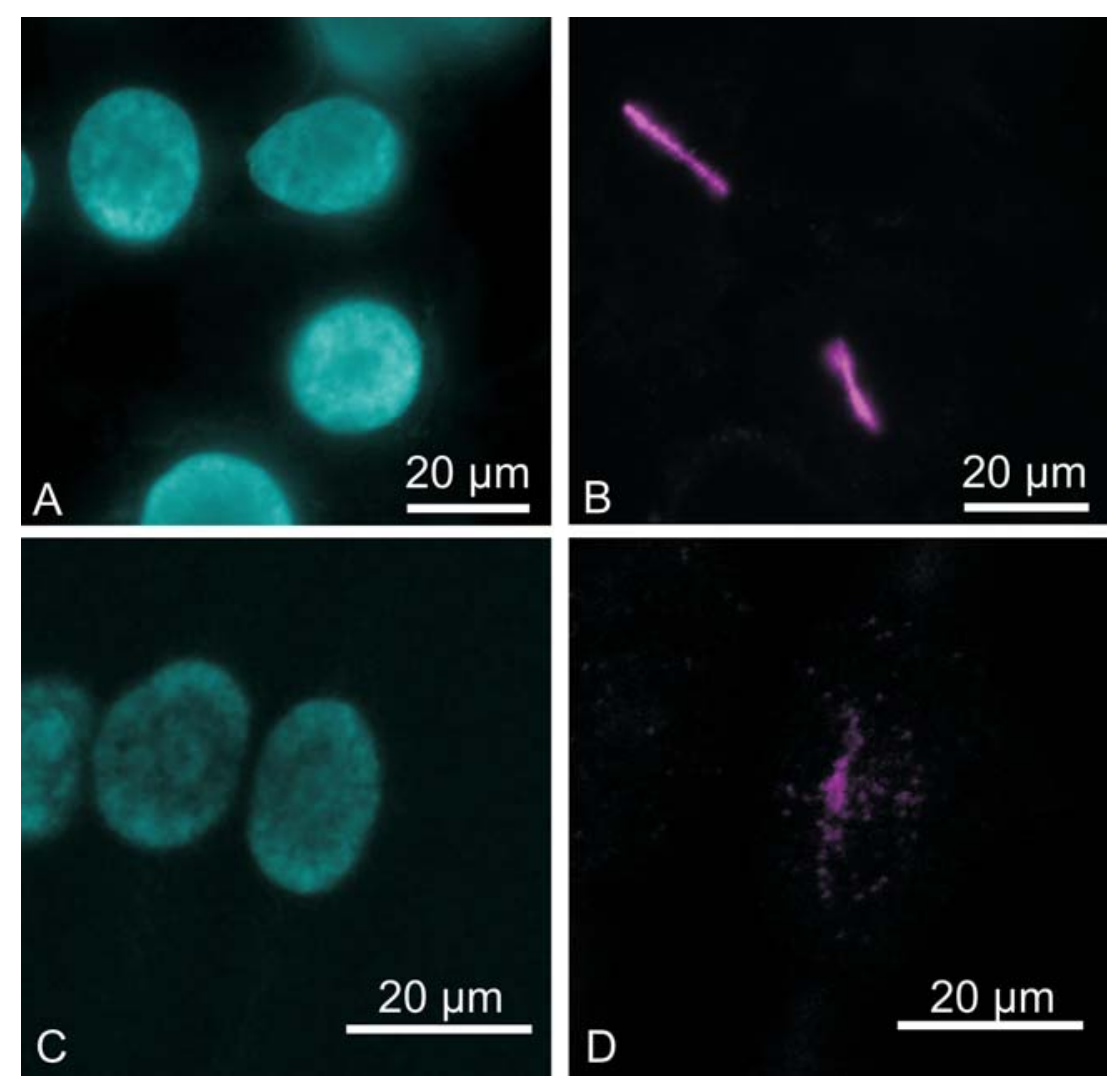

Figure 8 (A, B) Epifluorescence image of HeLa cell nuclei containing CPDs. (A), chromatin was counterstained with Hoechst 33342. (B), HeLa cell nuclei stained with $\alpha$-CPD-antibodies (Alexa 488). The irradiation conditions for CPD induction were: $12 \mathrm{~mW}$ average power at the sample, $\lambda_{\mathrm{C}}=773 \mathrm{~nm}$, $t_{\mathrm{P}}=183$ fs. The pixel dwell time was $25 \mathrm{~ms}$. The objective lens was a Zeiss oil immersion $40 \times$ EC-Plan-Neofluar N. A. 1.3. (C, D) Image of a HeLa cell nucleus labeled with $\alpha$-PAR-antibodies. Image $(\mathrm{C})$, chromatin staining with Hoechst 33342. Image (D), $\alpha$-PARantibody staining. Irradiation parameters: $P=16 \mathrm{~mW}, \quad \lambda_{\mathrm{C}}=1040 \mathrm{~nm}$, $t_{\mathrm{P}}=70 \mathrm{fs}$, pixel dwell time $32 \mathrm{~ms}$. 
induced by irradiation at this wavelength are cyclobutane pyrimidine dimers (CPDs). On the other hand, if cells are sensitized by incubation with 5-bromo-2'-deoxyuridine (BrdU) prior to irradiation, UV light can lead to DNA photolysis [31]. If sufficient photon density is provided, the same photochemical reactions may be triggered by two- or three-photon absorption of near-infrared light [32]. The advantage of infrared-triggered nonlinear photochemistry is the spatial confinement of the damage in all three dimensions. This feature is crucial for the study of DNA repair processes and the recruitment of repair proteins.

Figure 8(A, B) demonstrates the induction of CPDs in cellular DNA by three-photon absorption. The live cells were irradiated along a single line with $777 \mathrm{~nm}$ pulses from the Er:fiber laser with an average power at the sample of $12 \mathrm{~mW}$. The beam was focused by a Zeiss 40-EC-Plan-Neofluar 1.3 oil objective. The pulse duration was shorter than $200 \mathrm{fs}$ and the pixel dwell time $25 \mathrm{~ms}$. The cells were fixed immediately after irradiation and labeled with a monoclonal antibody specific for CPDs. The distribution of the damage along the path of the laser beam within the nuclei is clearly visible. The dimensions of the smallest damage we could induce were: $(600 \pm 10) \mathrm{nm}$ FWHM in the lateral direction and $(1.69 \pm 0.19) \mu \mathrm{m}$ FWHM in the axial direction, corresponding to a sub-femtoliter excitation volume.

Finally, in Figure 8(C, D) we have exploited sensitization with BrdU to induce DNA strand breaks with the NIR excitation tuned to $1040 \mathrm{~nm}$. The nuclei of live HeLa cells were irradiated along two lines crossing at a right angle in the middle of the nucleus. The average power at the sample was $16 \mathrm{~mW}$ and the pixel dwell time $32 \mathrm{~ms}$. The cells were fixed immediately after irradiation and DNA breaks were indirectly visualized by an antibody specific for poly(ADP-ribose). This is a posttranslational protein modification that is efficiently induced as a consequence of DNA strand breaks [33]. Foci of poly(ADP-ribose) are visible along the irradiated lines. The highest level of modification is observed around the crossing point of the two lines.

\section{Conclusion}

In this study, we have developed a versatile scanning microscope system equipped with an advanced femtosecond fiber laser and efficient dispersion management correcting for second- and third-order effects. As a result, sub-40 fs pulses are provided in the focal plane of an objective lens with high numerical aperture. The entire setup is robust in operation with excellent noise performance and negligible drift. These features are central in applications where high scan- ning rates and/or long illumination times are required. The available wavelength range is extended beyond $1 \mu \mathrm{m}$, a region that promises deeper sample penetration $[6,34]$ and lower photodamage as compared to excitation around $800 \mathrm{~nm}$. Therefore, our microscope has high potential for intravital applications. Despite its compactness, it provides sufficient power levels to perform 3D-confined photomanipulations in living cells, as demonstrated by the threephoton induction of UV-photoproducts and DNA strand breaks. Regarding linear fluorescence excitation, the central advantage of our microscope is continuous tunability in the visible range. Here, we demonstrate that by tuning the excitation wavelength, the irradiation conditions for fluorophores may be optimized in terms of photostability.

Acknowledgements The authors thank R. Meldrum for advice on three-photon induction of DNA damage, A. Benz and C. Strasser for immunostaining, and D. Hermann for expert technical assistance. We are indebted to A. Bürkle for providing the $\alpha$-PAR-antibody and to R. Tsien for mCherry and dTomato cDNA. Technical support by Carl Zeiss MicroImaging GmbH and Toptica Photonics AG is gratefully acknowledged. This study was supported by the Center for Applied Photonics of the University of Konstanz and by the German Research Foundation (grant DFG MA/2385/2-3 to EFM).

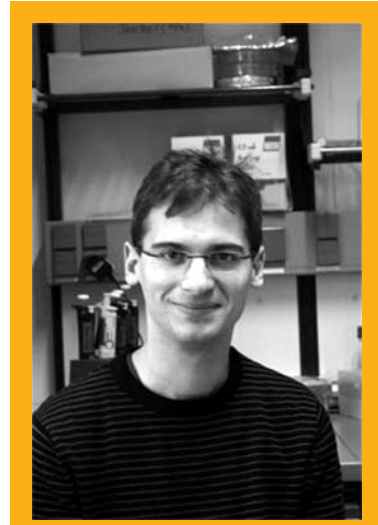

Daniel Traeutlein was born in Ravensburg, Germany, in 1980. He received his degree in Physics in 2003 from the University of Konstanz. $\mathrm{He}$ has started his $\mathrm{PhD}$ within the Center for Applied Photonics, at the University of Konstanz.

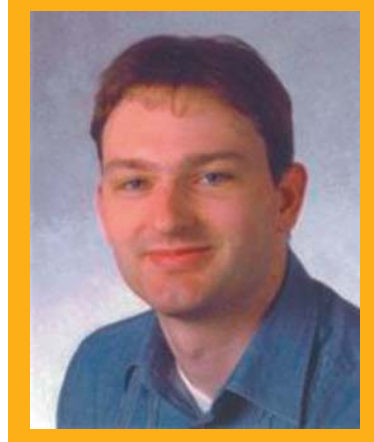

Florian Adler, born in 1978 in Wasserburg am Inn, Germany, received his Diploma in Physics in 2003 from the Technical University of Munich. He earned his PhD degree in 2007 from the University of Konstanz and is currently a Humboldt fellow and postdoctoral research associate at JILA, University of Colorado at Boulder. 


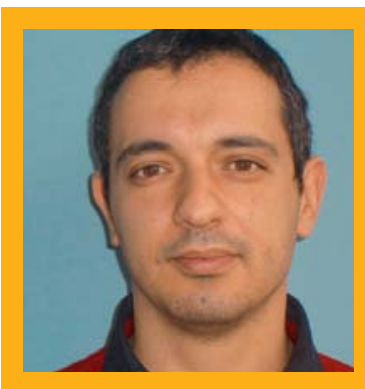

Konstantinos Moutzouris was born in Athens, Greece, in 1974. He received his degree in Physics from the National and Kapodistrian University of Athens, Greece, in 1998 and a $\mathrm{PhD}$ degree in Nonlinear Optics from the University of St. Andrews, UK, in 2003. His PhD thesis involved work on nonlinear frequency conversion in isotropic semiconductor waveguides.

In 2003, he moved for a short research visit to the Technical University of Munich, Germany and later the same year he joined the Chair of Modern Optics and Quantum Electronics at the University of Konstanz as a postdoctoral researcher. His work focused on femtosecond fiber-laser-based nonlinear frequency conversion devices operating in the spectral range between the ultraviolet and the near infrared. In 2006, he moved to the Federal Institute of Technology (ETH) Zurich, Switzerland, working towards the extension of these nonlinear devices into the mid-infrared. Konstantinos Moutzouris is an author or co-author of 30 papers in international journals and conference proceedings.

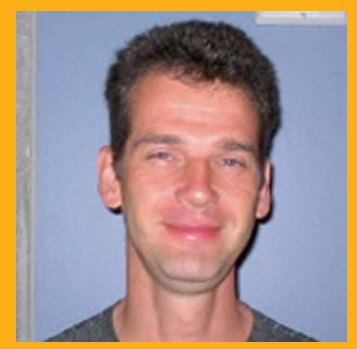

Andreas Jeromin was born in Hannover, Germany, and received his $\mathrm{PhD}$ in neuroscience from University of Toronto/Mt. Sinai Hospital in 2002. He obtained postgraduate training at Baylor College of Medicine in Houston, TX, before joining the University of Texas as research assistant professor. He joined the Allen Institute for Brain Science in Seattle, WA, in 2007 and is the current manager of the Methods Development department. Andreas Jeromin has been co-author on more than 60 publications in internationally recognized journals and has presented at various national and international meetings.

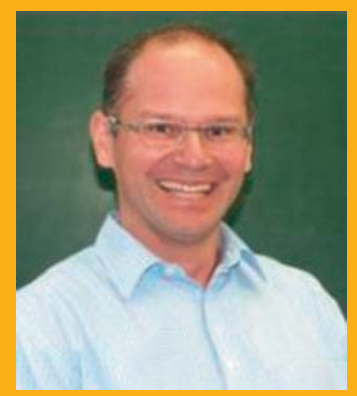

Alfred Leitenstorfer, born 1967, received his Diploma and $\mathrm{PhD}$ in Physics from the Technical University of Munich in 1993 and 1996, respectively. He worked as a postdoc in the Advanced Photonics Research Department of Bell Laboratories, Holmdel, from 1997 to 1998. In 2000, he finished his Habilitation in Experimental Physics and was faculty member at both the Technical University and the
Ludwig-Maximilians University of Munich in 2001 and 2002. Since 2003, he holds the Chair of Modern Optics and Quantum Electronics at the Department of Physics of the University of Konstanz and serves as Head of the Center for Applied Photonics. Alfred Leitenstorfer's research currently focuses on ultrafast quantum physics with solid-state nanostructures, quantum kinetics of low-energy excitations and optical phase control, as well as femtosecond technologies and their applications. An interactive publication list and more detailed information are accessible on the internet under www.uni-konstanz.de/quantum-electronics.

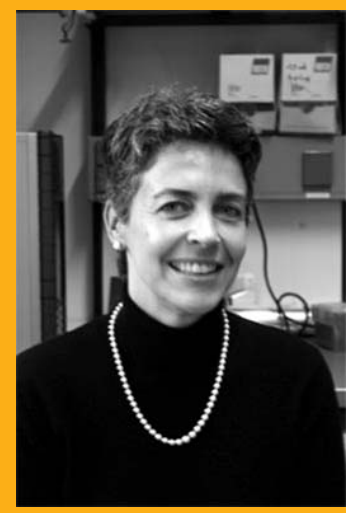

Elisa Ferrando-May, born in 1962, obtained a MSc in Biochemistry from the University of Tübingen and completed her Ph.D. at the Max-Planck Institute for Biochemistry in Martinsried in 1993. She joined the University of Konstanz as a postdoctoral research associate in Molecular Toxicology in 1996 where she became a Principal Investigator in 2001. In 2007 she was promoted head of the bioimaging core facility of the Dep. of Biology. Her current research interests include the development and application of novel imaging techniques to the study of nuclear processes in cell death, in particular chromatin alterations and nucleocytoplasmic transport.

\section{References}

[1] W. Denk, J. H. Strickler, and W. W. Webb, Science 248, 73-76 (1990).

[2] F. Helmchen and W. Denk, Nat. Methods 2, 932-940 (2005).

[3] N. C. Shaner, R. E. Campell, P. A. Steinbach, B. N. G. Giepmans, A. E. Palmer, and R. Y. Tsien, Nat. Biotechnol. 22, 15621-15626 (2004).

[4] D. Shcherbo, E. M. Merzlyak, T. V. Chepurnykh, A. F. Fradkov, G. V. Ermakova, E. A. Solovieva, K. A. Lukyanov, E. A. Bogdanova, A. G. Zaraisky, S. Lukyanov, and D. M. Chudakov, Nat. Methods 4, 741-746 (2006).

[5] T.-M. Liu, S.-W. Chu, C.-K. Sun, B.-L. Lin, P.-C. Cheng, and I. Johnson, Scanning 23, 249-254 (2001).

[6] I. Rimke, E. Büttner, V. Andresen, and P. Friedl, "Infrared multiphoton microscopy beyond 1 micron: system design and biomedical applications," Proc. SPIE 6442 (2007)

[7] G. McConnell, Phys. Med. Biol. 52, 717-724 (2007). 
[8] A. C. Millard, P. W. Wiseman, D. N. Fittinghoff, K. R. Wilson, J. Squier, and M. Müller, Appl. Opt. 38, 7393-7397 (1999).

[9] N. Deguil, E. Mottay, F. Salin, P. Legros, and D. Choquet, Microsc. Res. Technol. 63, 23-26 (2004).

[10] C. Dunsby, P. M. P. Lanigan, J. McGinty, D. S. Elson, J. Requejo-Isidro, I. Munro, N. Galletly, F. McCann, B. Treanor, B. Önfelt, D. M. Davis, M. A. A. Neil, and P. M. French, J. Phys. D: Appl. Phys. 37, 3296-3303 (2004).

[11] G. McConnell, Opt. Exp. 12, 2844-2850 (2004).

[12] J. A. Palero, V. O. Boer, J. C. Vijverberg, H. C. Gerritsen, and H. J. C. M. Sterenborg, Opt. Exp. 13, 53635368 (2005).

[13] J. R. Unruh, E. S. Price, R. G. Molla, L. Stehno-Bittel, C. K. Johnson, and R. Hui, Opt. Exp. 14, 9825-9831 (2006).

[14] T. Betz, J. Teipel, D. Koch, W. Härtig, J. Guck, J. Käs, and H. Giessen, J. Biomed. Opt. 10, 054009 (2005).

[15] J. Teipel, D. Türke, H. Giessen, A. Killi, U. Morgner, M. Lederer, D. Kopf, and M. Kolesik, Opt. Exp. 13, 1477-1482 (2005).

[16] F. Tauser, A. Leitenstorfer, and W. Zinth, Opt. Exp. 11, 594 (2003).

[17] The technology developed for this laser source is commercially available from TOPTICA Photonics AG.

[18] K. Moutzouris, F. Sotier, F. Adler, and A. Leitenstorfer, Opt. Exp. 14, 1905-1912 (2006).

[19] F. Tauser, F. Adler, and A. Leitenstorfer, Opt. Lett. 29, 516-518 (2004).
[20] K. Moutzouris, F. Adler, F. Sotier, D. Träutlein and A. Leitenstorfer, Opt. Lett. 31, 1148-1150 (2006).

[21] Z. Bor, J. Mod. Opt. 35, 1907-1918 (1988).

[22] Y. Barad, H. Eisenberg, M. Horowitz, and Y. Silberberg, Appl. Phys. Lett. 70, 922-924 (1997).

[23] W. R. Zipfel, R. M. Williams, and W. W. Watt, Nat. Biotechnol. 21, 1369-1377 (2003).

[24] S.-P. Tai, W.-J. Lee, D.-B. Shieh, P.-C. Wu, H.-Y. Huang, C.-H. Yu, and C.-K. Sun, Opt. Exp. 14, 61786187 (2006).

[25] S.-W. Chu, I.-H. Chen, T.-M. Liu, C.-K. Sun, S.-P. Lee, B.-L. Lin, P.-C. Cheng, M.-X. Kuo, and D.-J. Lin, J. Microsc. 208, 190-200 (2002).

[26] M. Müller, J. Squier, and G. J. Brakenhoff, Opt. Lett. 20, 1038-1040 (1995).

[27] R. Wolleschensky, T. Feurer, R. Sauerbrey, and U. Simon, Appl. Phys. B 67, 87-94 (1998).

[28] Donnert, C. Eggeling, and S. W. Hell, Nat. Methods 4, 81-86 (2007).

[29] C. Eggeling, A. Volkmer, and C. A. M. Seidel, ChemPhysChem 6, 791-804 (2005).

[30] A. Jeromin, L.-L. Yuan, A. Frick, P. Pfaffinger, and D. Johnston, J. Neurophysiol. 90, 2741-2745 (2003).

[31] C. L. Limoli and J. F. Ward, Radiat. Res. 134, 160169 (1993).

[32] R. A. Meldrum, S. W. Botchway, C. W. Wharton, and G. J. Hirst, EMBO reports 4, 1144-1149 (2003).

[33] A. Buerkle, BioEssays 23, 795-806 (2001).

[34] R. R. Anderson and J. A. Parrish, J. Invest. Dermatol. 77, 13-19 (1981). 